\title{
Effect of strain rate on shear properties and fracture characteristics of DP600 and AA5182-O sheet metal alloys
}

\author{
Taamjeed Rahmaan ${ }^{\mathrm{a}}$, Cliff Butcher, Armin Abedini, and Michael Worswick \\ University of Waterloo, Mechanical and Mechatronics Engineering Department, Waterloo, Ontario, Canada
}

\begin{abstract}
Shear tests were performed at strain rates ranging from quasi-static $\left(.01 \mathrm{~s}^{-1}\right)$ to $600 \mathrm{~s}^{-1}$ for DP600 steel and AA5182-O sheet metal alloys at room temperature. A miniature sized shear specimen was modified and validated in this work to perform high strain rate shear testing. Digital image correlation (DIC) techniques were employed to measure the strains in the experiments, and a criterion to detect the onset of fracture based on the hardening rate of the materials is proposed. At equivalent strains greater than 20\%, the DP600 and AA5182 alloys demonstrated a reduced work hardening rate at elevated strain rates. At lower strains, the DP600 shows positive rate sensitivity while the AA5182 was not sensitive to strain rate. For both alloys, the equivalent fracture strain and elongation to failure decreased with strain rate. A conversion of the shear stress to an equivalent stress using the von Mises yield criterion provided excellent agreement with the results from tensile tests at elevated strain rates. Unlike the tensile test, the shear test is not limited by the onset of necking so the equivalent stress can be determined over a larger range of strain.
\end{abstract}

\section{Introduction}

Advanced High Strength Steels (AHSS) and aluminum alloys are currently of great interest in the automotive industry due to their superior strength-to-weight ratio and adequate formability. Dual phase (DP) steels can be found in many vehicle structural components while 5000-series aluminum alloys are commonly used in the fabrication of inner body panels. In automobile crash events or high speed metal forming, the local strain rates can vary from quasi-static conditions to over $1000 \mathrm{~s}^{-1}$. Understanding the constitutive response and fracture mechanisms associated with dynamic loading is critical for the accurate design of sheet metal forming operations for structural components and their subsequent performance in vehicle crash simulations.

Sheet metal forming processes often involve large plastic strains. Optimization of the forming techniques requires an accurate knowledge of the elastoplastic behaviour of the metals until failure. Uniaxial tensile tests are used to characterize the mechanical properties of sheet metals until the onset of necking. However, diffuse or localized necking occurs in this test, limiting the accurate measurements of the behaviour of the materials to relatively small strains. Shear tests can investigate the material response until large plastic strains since localized necking does not occur and plane stress conditions are satisfied until fracture. In addition, the development of stress statedependent fracture surfaces and damage models to predict failure in automotive forming and crash simulations has created great interest in developing experimental tests to characterize failure under shear stress states $[1,2]$.

Unlike tensile tests, obtaining the shear stress of sheet metals is difficult as the shear stress distribution

\footnotetext{
${ }^{a}$ Corresponding author: trahmaan@uwaterloo.ca
}

is generally inhomogeneous and there is no intrinsic gauge length that can define the local strain. Torsion tests are not suitable for sheet materials; therefore, various simple shear test geometries have been developed [3-8]. The most common shear test involves clamping a rectangular specimen and moving the clamps in opposite directions to impose a shear deformation. Alternatively, various experimental techniques and geometries have been proposed in the literature but most require a complex geometry that is inconvenient for dynamic testing [9-12].

Recently, Peirs et al. [12] developed a simple shear specimen geometry for both quasi-static and dynamic strain rates using numerical optimization and digital image correlation (DIC) to obtain the local shear strain. The design of this geometry is an evolution of the geometries proposed by Tarigopula et al. [13] and Bao and Wierzbicki [14]. The advantage of this geometry is that it is relatively simple to fabricate, does not require through-thickness machining and provides a uniform shear strain distribution that can be used to resolve the shear stress response of the material. Recent work by Abedini et al. [15] has demonstrated the advantages of the Peirs et al. [12] geometry for material characterization.

In the present work, the specimen geometry developed by Peirs et al. [12] has been modified in order to conduct experiments utilizing the testing apparatus with smaller grip size. The shear deformation behaviour and fracture characteristics of DP600 and AA5182-O sheet metal alloys have been investigated at quasi-static, 10,100, and $600 \mathrm{~s}^{-1}$ strain rates. The dynamic shear tests served as a companion study to the recent high rate tensile characterization of these materials by the authors [16]. A method is proposed to determine the fracture strain based on hardening curve of the material. 


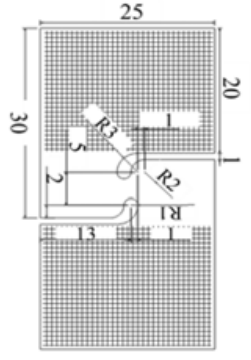

a

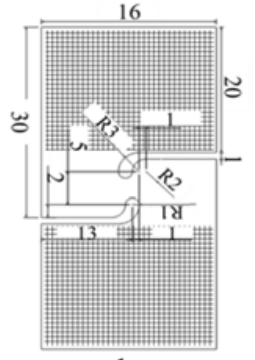

b

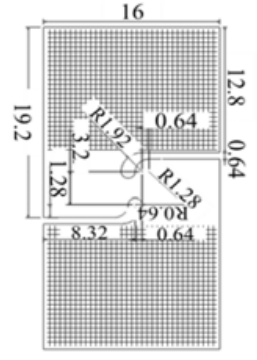

c
Figure 1. Shear specimen geometry: (a) regular size [12] (b) trimmed and (c) mini (scaled down) specimen. Dimensions are in $\mathrm{mm}$. Shaded regions are the grip area.

\section{Experiments}

\subsection{Materials}

Materials studied were DP600 and AA5182-O in nominal $1.5 \mathrm{~mm}$ thick sheets. They were selected due to their importance in lightweight automotive applications and their difference in observed rate sensitivity. Specimens were tested along the sheet rolling direction. The reader is referred to Rahmaan et al. [16] for the detailed mechanical properties and tensile behaviour for a wide range of strain rates.

\subsection{Specimen geometry}

The specimen geometry developed by Peirs et al. [12] was used in this work for the quasi-static shear testing. However, the width of the specimen was larger than the grip slot sizes of the dynamic strain rate experimental apparatus available at University of Waterloo. Thus, the shear specimen geometry developed by Peirs et al. [12] (referred to as regular shear specimen herein) was modified in two phases. In the first phase, the width of the regular shear specimen was trimmed down to match the size of the grip slots of the apparatus, while all other dimensions of the regular shear specimen were kept unchanged. In the second phase, all the dimensions of regular size specimen were scaled down by $36 \%$, (referred to as mini shear specimen). The geometries of the three shear specimens are shown in Fig. 1.

\subsection{Apparatus and procedure}

An Instron model 1331 servo-hydraulic testing machine was used to conduct shear testing at nominal strain rates of $0.01 \mathrm{~s}^{-1}$ (or quasi-static). Cross-head velocities for the experiments were $0.03 \mathrm{~mm} / \mathrm{s}$ for the regular and trimmed size shear specimen testing, and $0.019 \mathrm{~mm} / \mathrm{s}$ was used for the mini size shear specimen testing.

A Hydraulic Intermediate Strain Rate (HISR) apparatus developed at the University of Waterloo was used to perform shear tests at strain rates of 10, 100, and $600 \mathrm{~s}^{-1}$. The reader is referred to [17] for a detailed description of the HISR apparatus. Cross-head velocities of approximately $35 \mathrm{~mm} / \mathrm{s}, 370 \mathrm{~mm} / \mathrm{s}$, and $2100 \mathrm{~mm} / \mathrm{s}$ were used to achieve the nominal strain rates of 10,100, and $600 \mathrm{~s}^{-1}$, respectively.

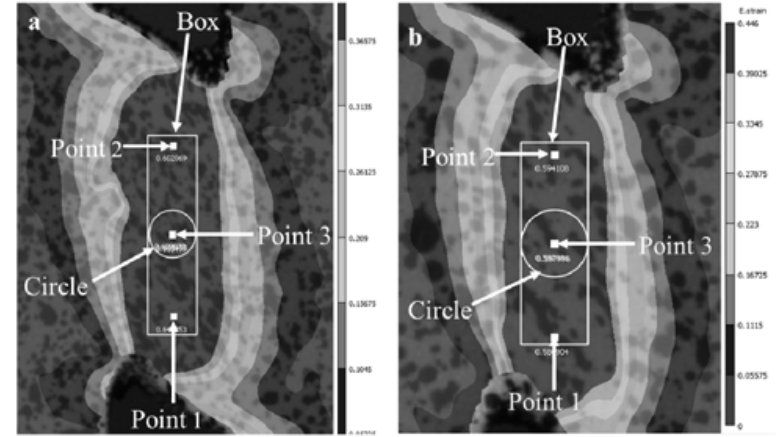

Figure 2. Different inspector tools of the DIC data processor software, Vic-2D positioned on the deformed DP600 shear specimens for a quasi-static strain rate test condition using (a) regular size [12] and (b) mini size specimen.

The strains were measured using digital image correlation (DIC) techniques. A Point Grey Research GRAS-50S5M-C camera was employed for the quasistatic condition and a Photron SA5 high speed camera for the higher strain rates. In the present work, the frame rates used were $6 \mathrm{fps}, 2,000 \mathrm{fps}, 17,500 \mathrm{fps}$, and $40,000 \mathrm{fps}$ for nominal strain rates of $1,10,100$, and $600 \mathrm{~s}^{-1}$, respectively. Full-field logarithmic (true) strain measurements were obtained using Vic-2D software from Correlated Solutions Inc. using the incremental correlation option to account for severe local strains. As discussed in Peirs et al. [12] and Abedini et al. [15], the average shear stress, $\tau_{\text {avg }}$, is computed as:

$$
\tau_{\text {avg }}=\frac{F}{A_{\text {shear }}}=\frac{F}{L t}
$$

where $F$ is the force, $t$ is the thickness, and $L$ is the length of the shear region with nominal values of $1.9 \mathrm{~mm}$ for the mini-shear sample and $3.0 \mathrm{~mm}$ for the other geometries. The as-fabricated length and thickness of each sample was measured using an optical microscope. Three to six repeat tests were performed for each test condition and an average stress-strain curve was generated by interpolating the test data using Von Mises equivalent strain increments of 0.002 .

\section{Results and discussion}

\subsection{Shear stress-strain behaviour}

Due to the small sample size and severe local strains within the gauge region it is instructive to investigate the procedure for extracting the local strain measurements. The local strain measurements can be extracted from single points (highly variable and noisy) or averaged over a user-defined area (rectangular or circular) which introduces a length scale into the analysis. To establish a consistent methodology for the DIC analysis of the shear samples, three different inspector tools were considered: a rectangular box $(0.3 \times 1.5 \mathrm{~mm})$, a circle with a radius of $0.3 \mathrm{~mm}$, and 3 points taken within the shear band as shown in Fig. 2 for a DP600 sample. The resulting shear stress vs. equivalent strain response obtained using the three measurement techniques is shown in Fig. 3 and 


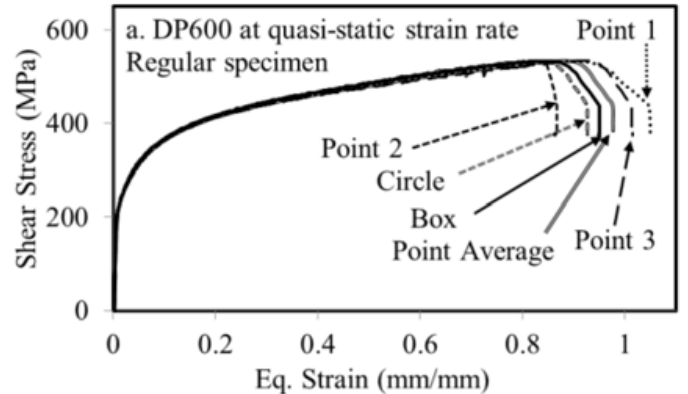

Figure 3. Quasi-static shear stress response of DP600 extracted using different inspector tools.

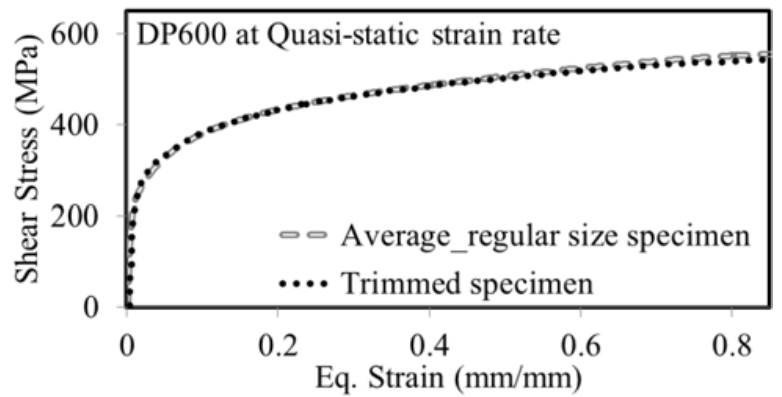

Figure 4. Comparison of the quasi-static shear stress responses of DP600 obtained using the regular and trimmed specimens.

show good agreement until very large strains on the order of $80 \%$ for DP600. The fracture strain computed using these different methods shows some dependency upon the measurement techniques with the point measurements showing some variation while the box and circle inspector tools give similar strains. The point measurements are inherently noisy so an average of the three points within the shear zone gives a very similar measurement as the area-averaged values. Thus, it would be safe to conclude that either the rectangular box or circular inspector tool provides accurate data as long as they are positioned within the shear region of the specimen.

Although the regular size shear specimen was successfully implemented for high strain rate testing by Peirs et al. [12], it was not possible to utilize this geometry for high strain rate testing due to narrow grip sizes of the current apparatus. In order to utilize the shear specimen in the existing dynamic strain rate testing apparatus, the width of the specimen was trimmed down from $25 \mathrm{~mm}$ to $16 \mathrm{~mm}$, while all other dimensions remained the same. The shear stress vs. equivalent strain data for DP600 sheet material obtained using the trimmed specimen is presented in Fig. 4 and compared with the data from regular size shear specimen. The data obtained from both specimens are found be in excellent agreement. However, as shown in Fig. 5, the trimmed specimen does not have sufficient material in the grips to withstand an induced rotation due to severe shear deformation within the gauge section. This shear-induced rotation did not have an apparent effect on the stress-strain curves at quasi-static conditions but it could possibly introduce a misalignment and errors in a high rate shear test using a tensile split Hopkinson bar. As such, the trimmed sample geometry was discarded.

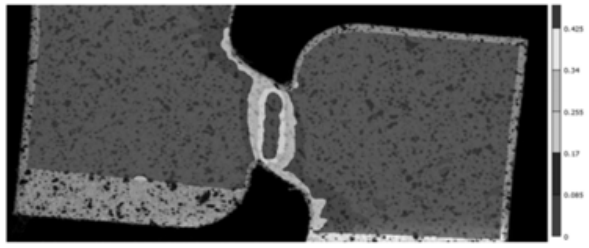

Figure 5. Equivalent strain distribution of the trimmed DP600 shear specimen prior to fracture.

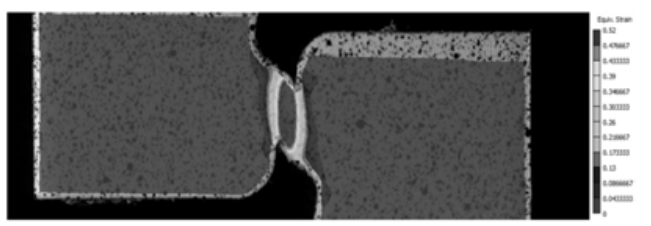

Figure 6. Strain contour plot of plastically deformed DP600 mini specimen at quasi-static strain rate test condition.

The regular size shear sample was then scaled down by $36 \%$ to obtain a sample width of $16 \mathrm{~mm}$ and is referred to as the mini-shear sample. The small dimensions of the new sample required fabrication using electric discharge machining. As shown in Fig. 6, the shear-induced rotation was removed and the same shear stress response was obtained for both the DP600 and AA5182 as shown in Figs. $7 \mathrm{a}$ and $7 \mathrm{~b}$, respectively. The experimental data obtained with the regular and mini-shear specimens is in excellent agreement at quasi-static conditions with very good repeatability. Thus, it is assumed that the mini-shear specimen geometry is suitable for dynamic shear tests as well since the regular sample was proven successful by Peirs et al. [12]. Different DIC strain inspector tools were also investigated for the miniature shear specimen with the same trends the regular specimen. A rectangular $(0.2 \times 0.7 \mathrm{~mm})$ or small cicle with a radius of $0.2 \mathrm{~mm}$ placed within the shear zone gives consistent strain measurements.

The effect of strain rate on shear stress versus equivalent strain for DP600 and AA5182-O sheet materials is shown in Figs. 8a and 8b, respectively. For DP600, the yield stress scales upwards with increasing strain rate, while the shape of shear stress curve (or the hardening behavior) decreases with the increase of strain rate. As expected, the yield stress of AA5182-O is found to be strain rate insensitive; however, the hardening behavior of the material shows negative rate sensitivity.

The maximum temperature rise, $\Delta T$ using an adiabatic heating assumption was calculated using Eq. (2), where $\beta$ is the ratio of plastic work converted into heat (taken as 0.9), $\rho$ is the material density, $C_{p}$ is the heat capacity of the material, and the remainder is the integral of the equivalent stress versus strain curve which is calculated as the area under the curve up to fracture strain. The adiabatic heat rise up to the fracture strain was calculated to be approximately $144{ }^{\circ} \mathrm{C}$ and $76^{\circ} \mathrm{C}$ at $600 \mathrm{~s}^{-1}$ for DP600 and AA5182-O, respectively.

$$
\Delta T=\frac{\beta}{\rho \cdot C_{p}} \int \sigma d \varepsilon .
$$



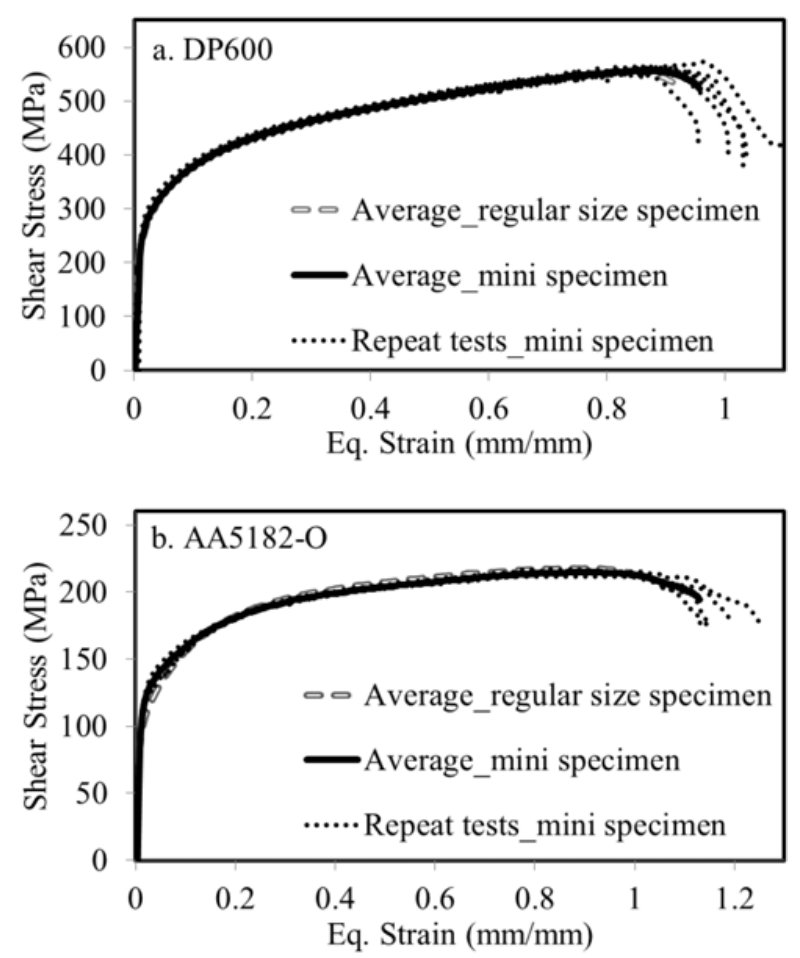

Figure 7. Comparison of the quasi-static shear stress response between the regular and mini-shear specimens for (a) DP600 and (b) AA5182-O.

The DP600 has positive rate sensitivity at strains up to approximately $30 \%$ and then the hardening rate becomes lower than quasi-static. AA5182-O is strain rate insensitive at strains up to approximately $25 \%$, while the hardening rate decreases as the strain rate increases from quasistatic to $10 \mathrm{~s}^{-1}$. The hardening rate of AA5182-O is rate insensitive for strain rates above $10 \mathrm{~s}^{-1}$. The observed change in hardening behavior of the materials is thought to be due to either local temperature rise or strain localization within the microstructure leading to damage and failure. Understanding of such behavior requires further experimental analysis and therefore was left for future research.

\subsection{Fracture criterion}

The onset of fracture in a shear test is difficult to determine visually since the cracks do not appreciably open. The cracks propagate through the material with the two fracture surfaces maintaining contact. Visual means of detecting fracture are further complicated by the painted surface required for the DIC analysis. As shown in Fig. 9, shear cracks are evident on the unpainted AA5182-O sample while the cracks are difficult to discern in painted samples. In order to increase the accuracy of the fracture strain data, a fracture criterion for the experimental data set is proposed herein based on the hardening rate.

The logarithmic (true) finite strain tensor for simple shear deformation maintains a plane strain loading condition at all times and the thickness remains constant. The modified maximum force criterion (MMFC) of Hora et al. [18] is an extension of the Considere criterion and states that the strain path in tensile-dominated stress states
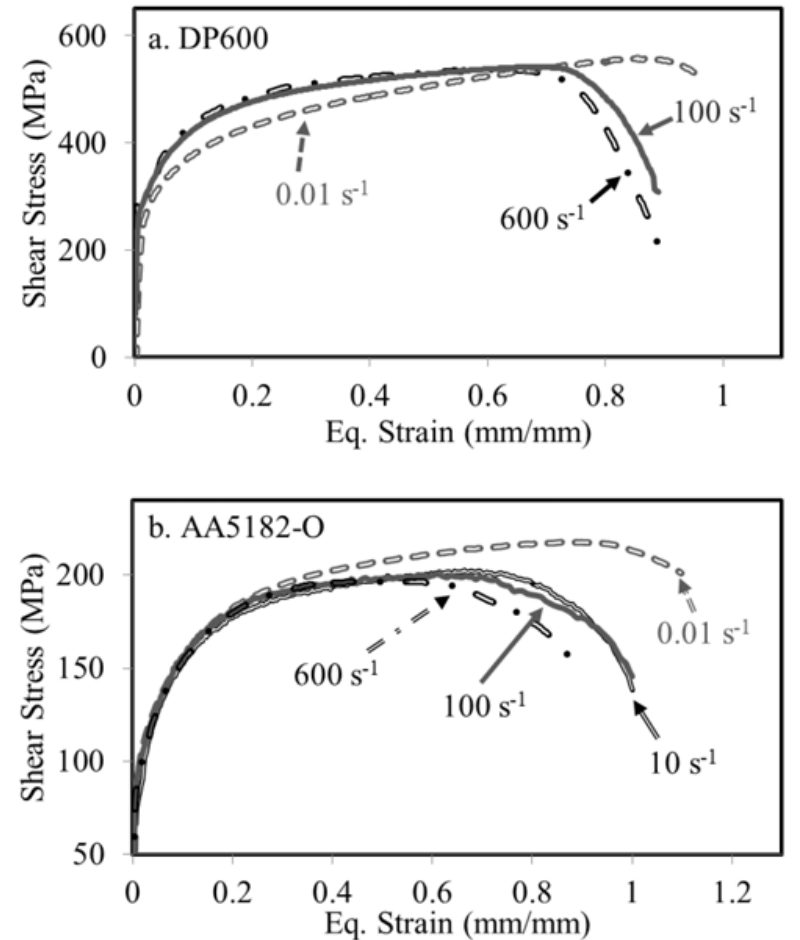

Figure 8. Averaged shear stress vs. equivalent strain curves for (a) DP600 and (b) AA5182-O sheet specimens at room temperature and strain rates from quasi-static to $600 \mathrm{~s}^{-1}$.

will converge to plane strain loading before fracture. As a material begins to lose its ability to work harden, the stress state will transition to plane strain and then abruptly fracture. Since the simple shear test is already in a constant state of plane strain, the loss of work hardening should coincide with the onset of cracking and local failure. This criterion can be expressed as

$$
\text { Hardening rate }=d \tau / d \varepsilon_{e q} \leq 0 \text { for fracture onset }
$$

where, $\tau$ is the average shear stress and $\varepsilon_{e q}$ is the von Mises equivalent strain.

Figure 10a shows the stress response and hardening rate along with the onset of fracture using Eq. (3). The onset of fracture is taken at the strain level when Eq. (3) is satisfied and remains negative for the duration of the test. Shear fracture is not immediate for AA5182-O and DP600 as they have moderate ductility and sufficient crack resistance so that the load decreases during crack propagation. An inspection of the local equivalent strain rate in Fig. 10b also shows an increase in the local strain rate that is relatively in good agreement with onset of cracking by using Eq. (3). As such, Eq. (3) provides a very good estimate for the onset of fracture.

It is important to note that the fracture criterion in Eq. (3) is not required for materials with relatively low ductility that are susceptible to shear fracture. Abedini et al. [15] observed that shear fracture of ZEK100 magnesium and the higher strength DP780 exhibited abrupt shear fracture without a decrease in the load prior to failure.

The fracture strain of DP600 and AA5182-O were obtained with respect to the strain rates from the 


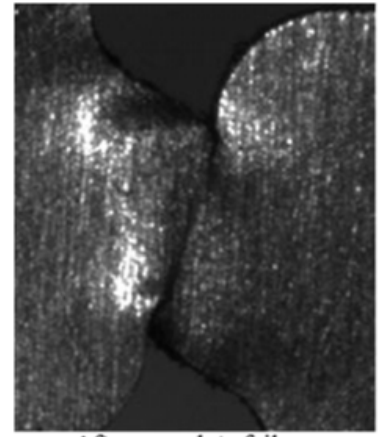

After complete failure Specimen without speckle

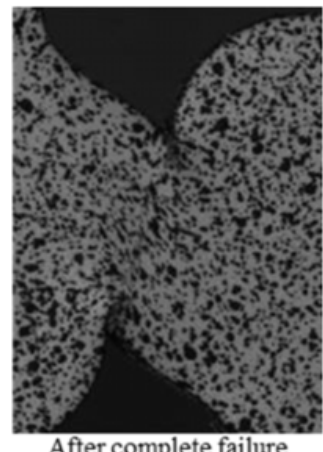

After complete failure Specimen with speckle
Figure 9. Fractured AA5182-O shear specimen with and without a painted speckle pattern for DIC strain analysis.
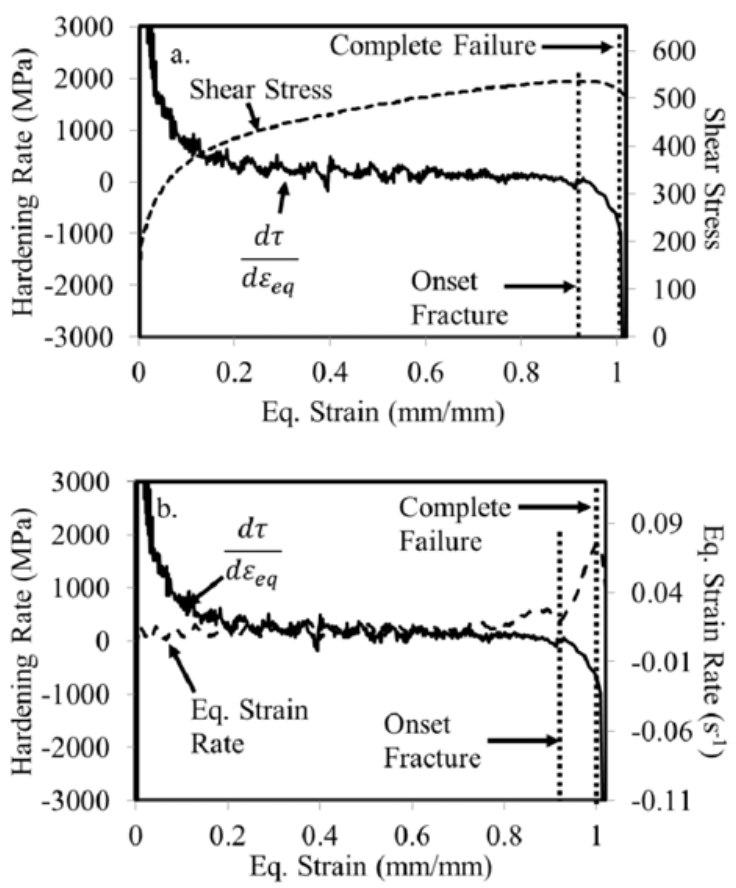

Figure 10. (a) Hardening rate and shear stress and (b) hardening rate and equivalent strain rate plotted against the equivalent strain indicating the onset fracture strain and a spike in strain rate at onset fracture for DP600 at a quasi-static strain rate.

experimental data based on the proposed fracture criteria. The effect of strain rate on the fracture strain for DP600 and AA5182-O sheet material is shown in Fig. 11a and Fig. 11b, respectively. Total elongation to fracture is also plotted against the strain rate in the same figures. A negative rate sensitivity trend is observed in the fracture strain and elongation to fracture for both materials. For an increase in strain rate from 0.01 to $600 \mathrm{~s}^{-1}$, the corresponding decrease in the equivalent fracture strain was found to be 0.25 and 0.33 for DP600 and AA5182$\mathrm{O}$ sheet material, respectively.

\subsection{Tensile and shear stress comparison}

A conversion of shear data to tensile data can be useful to obtain flow stress curves for larger strains as well as to
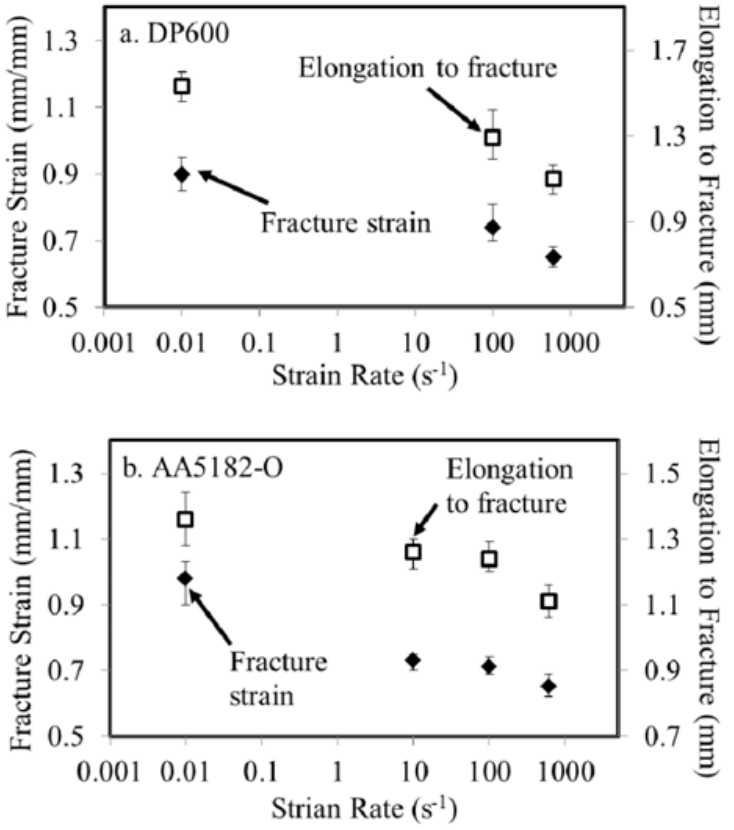

Figure 11. Equivalent fracture strain and elongation to fracture plotted against strain rates for (a) DP600 and (b) AA5182-O.

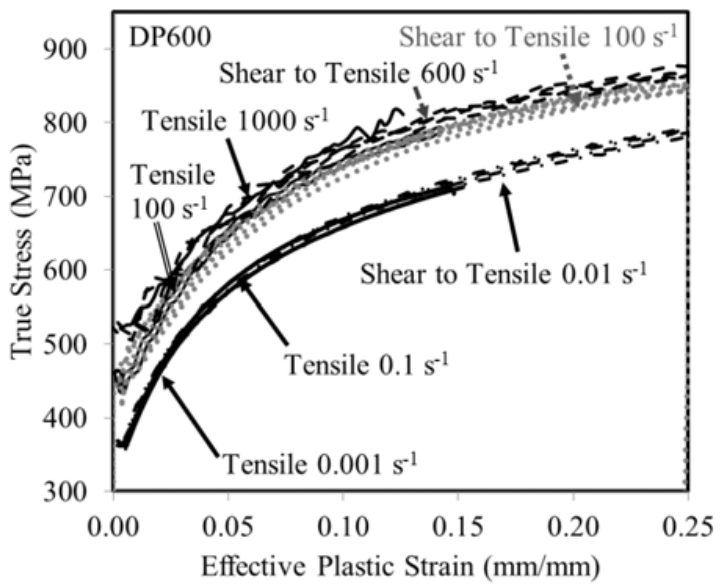

Figure 12. True stress vs. effective plastic strain comparison for experimental data from tensile [16] and shear testing at the strain rate ranging from 0.01 to $600 \mathrm{~s}^{-1}$.

obtain flow stress curves for ultra-high $\left(>10^{4} \mathrm{~s}^{-1}\right)$ strain rate testing conditions that are not typically achievable in the uniaxial tensile testing. The shear stress data obtained in this work was converted to tensile stress using Von-Mises criteria assuming isotropic material behaviour. Shear stress up to $30 \%$ equivalent strain was converted to equivalent tensile stress in order to stay within the infinitesimal strain regime.

The converted shear to tensile data is compared with the experimental data obtained from uniaxial tensile testing for the same sheet materials along diagonal $\left(45^{\circ}\right.$ to the rolling) direction [16] at comparable nominal strain rates (Figs. 12 and 13). Due to the relatively isotropic behaviour of DP600 sheet, the converted tensile data form shear testing shows an excellent agreement with the uniaxial tensile test data for strain rates ranging from low to high 

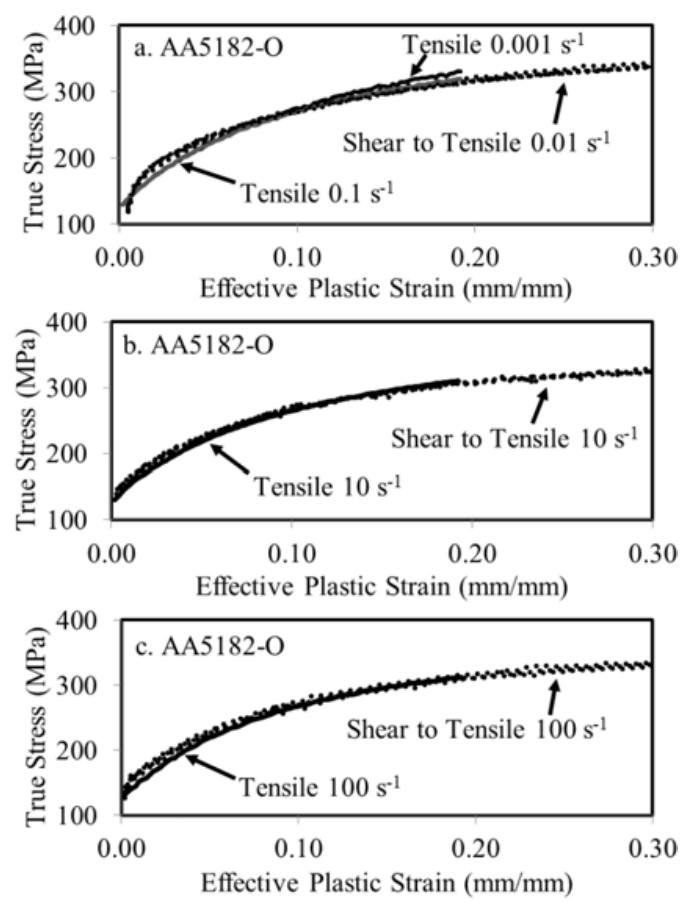

Figure 13. True stress vs. effective plastic strain comparison for experimental data from tensile [16] and shear testing at (a) quasistatic (b) 10 and (c) $100 \mathrm{~s}^{-1}$ strain rate.

(as shown in Fig. 12). Figure 13(a-c) compares tensile data converted from shear test data with the tensile data obtained from uniaxial tensile tests for AA5182-O. The tensile data converted from the shear data using the von Mises yield criterion shows very good agreement with each other at the dynamic strain rate test conditions for AA5182-O, while only a satisfactory comparison is observed at the quasi-static strain rate condition. Further improvement in converting shear data to tensile data could be achieved using anisotropic yield criteria such as Barlat anisotropic material models [19]. Future work will focus on ultra-high strain rate experiments using the mini-shear specimen on a Hopkinson bar to obtain the tensile flow stress response of these materials.

\section{Conclusions}

I. The shear specimen developed by Peirs et al. [12] was successfully modified to a mini size shear specimen in order to perform high strain rate shear testing.

II. At equivalent strains lower than approximately 0.25 , DP600 exhibited positive rate sensitivity, while AA5182-O is found to be strain rate insensitive. However, the hardening rate decreased at larger strains for both materials as the strain rate increased.
This reduction in hardening behavior is thought to be due to thermal softening at higher strain rates.

III. For an increase in strain rate from 0.01 to $600 \mathrm{~s}^{-1}$, the corresponding decrease in fracture strain was found to be approximately 0.25 and 0.33 for DP600 and AA5182-O sheet metals, respectively.

The authors greatly appreciate financial support from Automotive Partnerships Canada (APC), the Natural Sciences and Engineering Research Council (NSERC) of Canada, the Canada Research Chairs Secretariat, the Ontario Research Fund, Ford Research and Advanced Engineering, Amino Corporation, and ArcelorMittal.

\section{References}

[1] Y. Bai, T. Wierzbicki, Int. J. Impact Eng, 24, 10711096 (2008)

[2] Y. Bai, T. Wierzbicki, Int. J. Fracture, 161, 1-20 (2010)

[3] N. Iosipescu, J. Mater, 2, 537-566 (1967)

[4] E.F. Rauch, J. Eng. Mater. Tech, 131, 0111107-1-8 (2009)

[5] M. Gasperini, C. Pinna, W. Swiatnicki, Acta Mater, 40, 4195-4208 (1996)

[6] S. Bouvier, H. Haddadi, P. Levee, C. Teodosiu, J. Mater. Tech, 172, 196-103 (2006)

[7] J.R. Klepaczko, Int. J. Impact Eng, 15, 25-39 (1994)

[8] J.R. Klepaczko, A. Rusinek, Int. J. Plasticity, 17, 87-115 (2001) F. Gao, L. Gui, Z. Fan, Exp. Mech, 51, 891-901 (2011)

[9] A. Reyes, M. Eriksson, O.G. Lademo, O.S. Hopperstad, M. Langseth, Mat. And Design, 30, 596-608 (2009)

[10] J. Kang, D.S. Wilkinson, P.D. Wu, M. Bruhis, M. Jain, J.D. Embury, R.K. Mishra, J. Eng. Mater. Tech, 130, 1-5 (2008)

[11] J. Peirs, P. Verleysen, J. Degrieck, Exp. Mech, 52, 729-741 (2012)

[12] J. Peirs, P. Verleysen, W. Van Paepegem, J. Degrieck, Int. J. Impact Eng, 38, 406-415 (2011)

[13] V. Tarigopula, O.S. Hopperstad, M. Langseth, A.H. Clausen, F. Hild, O.G. Lademo, M. Eriksson, Exp. Mech, 48(2), 181-196 (2008)

[14] Y.B. Bao, T. Wierzbicki, Int. J. Mech. Sci, 46(1), 81-98 (2004)

[15] A. Abedini, C. Butcher, D. Anderson, M.J. Worswick, T. Skszek, SAE World Congress, Paper \# 2015-15H-0274 (2015)

[16] T. Rahmaan, A. Bardelcik. J. Imbert, C. Butcher, M. Worswick, submitted to Int. J. Impact Eng (2014)

[17] A. Bardelcik, Ph.D. thesis, Uni. of Waterloo (2012)

[18] P. Hora, L. Tong, J. Reissner, Proceedings of the Numisheet'96 Conference, 252-256 (1996)

[19] F. Barlat, Advanced Methods Mat. Forming, Springer Berlin Heidelberg, 1-18 (2007) 